\title{
CALIDAD DEL CUIDADO DEL ADULTO MAYOR EN ESCENARIO NO INSTITUCIONALIZADO
}

\author{
M.Ed.Olga Lidia Banda González, M.C.Cinthya Patricia Ibarra González \\ MCE.Ma. Guadalupe Vázquez Salazar, MSP.Laura Roxana de los Reyes Nieto \\ Alumnas del Doctorado en Enfermería, Cultura de los cuidados, de la \\ Universidad de Alicante, España. Profesoras de la Facultad de Enfermería campus \\ CD. Victoria, de la Universidad Autónoma de Tamaulipas, México.
}

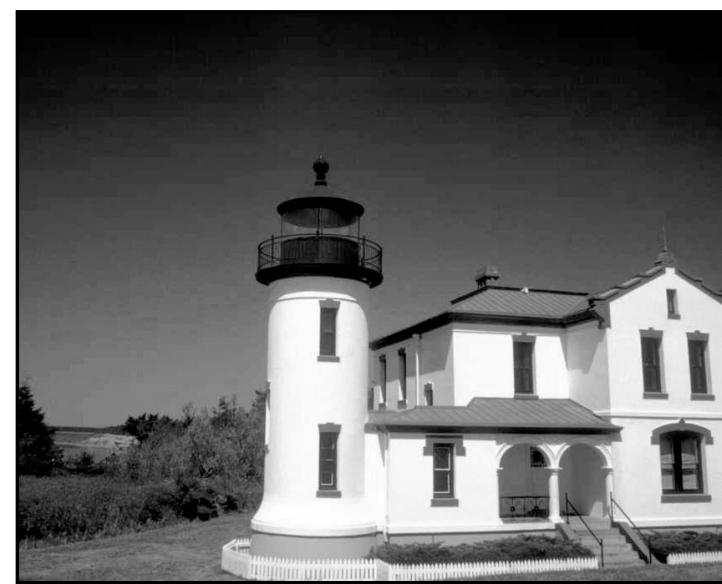

QUALITY OF CARE IN ADULTS AGED OVER 60 IN STAGES OTHER THAN THEIR OWN HOME

\section{ABSTRACT}

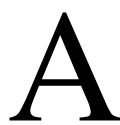

t present, human beings get more and more aged in a better estate of health, but the

consequence of that fact implies wider possibilities of being submitted to more chronic illnesses and disabilities, (Bazo, 1998).

In this respect, Lehr (1993) states that the hope for a good life not only means a longer life but a better quality of life. It is not how old a person could get but how he gets old, how does he age. Thus, the adult should be given better care in order to keep him in a good health condition with an interesting quality of life.

A research was done to discover the quality of care an adult gets when he lives in a different stage of his own home, his own environment, in a "Home". A formal, observational, descriptive, transversal study was run where the unit to be analyzed was an adult between 60 and 75 years of age.

Results showed that care provided to an aged adult in a stage other than his own home only contributes either to maintain his physical capacities or to perform his daily life activities, which in our opinion is not enough to be considered as "a quality of life" care.

Keywords: Aged adult, care, quality of life.

\section{RESUMEN}

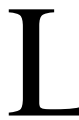

as personas en la actualidad están alcanzando cada vez edades más avanzadas en mejor estado de salud. Pero precisamente el hecho de que se viva mas conlleva a mayores probabilidades de presentar enfermedades crónicas e invalidantes (Bazo, 1998)

Al respecto Lehr (1993) menciona que la esperanza de vida no solo quiere decir longitud de vida, si no también la calidad de la misma. No solamente cuenta que tan vieja puede ser una persona, si no también como envejece; por lo que es necesario proporcionar al adulto mayor cuidados para mantenerlo en buenas condiciones y mejorar su calidad de vida.

Por lo anterior se investigó la calidad del cuidado de adulto mayor en escenario no institucionalizado, mediante un estudio observacional-descriptivo-transversal, donde la unidad de análisis fue el adulto de 60 a 75 años de edad.

Los resultados obtenidos demuestran que el cuidado proporcionado al adulto mayor en escenario no institucionalizado, solo contribuye al mantenimiento de sus capacidades físicas o ayudarle a realizar actividades de la vida diaria; sin embargo 
no podemos afirmar que esto contribuya a su calidad de vida.

Palabras clave: Adulto mayor, cuidado, calidad de vida

\section{INTRODUCCIÓN}

Las personas en la actualidad están alcanzando cada vez edades más avanzadas en mejor estado de salud. Pero precisamente el hecho de que se viva más conlleva a mayores probabilidades de tener enfermedades crónicas e invalidantes (Bazo, 1998).

La mayoría de las personas pueden tener la esperanza de llegar a una vejez avanzada, ya que de acuerdo con Papalia, Wendkos y Duskin (2001), desde 1955 en el mundo la expectativa de vida media se ha elevado el $37 \%$ esto debido a constantes avances de la ciencia que permiten conservar la vida durante un tiempo más prolongado, mismo que ha despertado una especial preocupación tanto en países de primer mundo como aquellos en vías de desarrollo

En México, de acuerdo con Rubalcava (1999), la esperanza de vida permitirá que para el año 2050 los ciudadanos alcancen los 84 años de edad, después de que en el año 1930 apenas se tenía una esperanza de vida de 36.2 años, lo cual habla de la necesidad de contar con políticas especiales para atender el tema del envejecimiento en nuestro país que aseguren la calidad de vida a la que pueden aspirar los adultos mayores sin ser marginados o tratados discriminatoriamente por la concepción generalizada que se tiene de ellos.

Delimitar cuando una persona pasa de la etapa de la madurez a la senectud, se acepta como inicio de la vejez los 60 años de edad, ya que la Asamblea Mundial del Envejecimiento, realizada por la ONU en 1982, delimitó a los diferentes grupos de personas que existen en la sociedad designando los términos de vejez, tercera edad, ancianos y adultos mayores a los individuos que alcanzan y rebasan las seis décadas.

En este sentido Lehr (1993) refiere que la esperanza de vida no sólo quiere decir la longitud de la vida, sino también, la calidad de la misma. No solamente cuenta qué tan vieja puede ser una persona, sino cómo envejece.
Ruiz y Quintero (1999) argumentan que el bienestar subjetivo o la satisfacción con la vida en la vejez, es el principal criterio de un envejecimiento exitoso. En la discusión actual acerca de este concepto, algunos autores han planteado que hay índices objetivos más que subjetivos, que deben utilizarse. Uno de estos índices objetivos es la longevidad; otro es el mantenimiento de la competencia para vivir en forma independiente; es decir, la calidad de vida.

En este sentido la calidad de Vida (CV) en la tercera edad debe estar ajustada a la esperanza de vida, de lo contrario aumentaría la expectativa de incapacidad. Por lo que matemáticamente se puede expresar que el aumento de la $\mathrm{CV}$ es inversamente proporcional a la expectativa de incapacidad (Camejo, Álvarez, Pérez \& Sariego, s/f).

La OMS propuso, en 1994, la siguiente definición para lograr el consenso de calidad de vida: "Percepción personal de un individuo de su situación en la vida, dentro del contexto cultural y de valores en que vive, y en relación con sus objetivos, expectativas, valores e intereses". Es un concepto extenso y complejo que engloba la salud física, el estado psicológico, el nivel de independencia, las relaciones sociales, las creencias personales y la relación con las características sobresalientes del entorno.

Aunque es imposible detener el paso de los años y el deterioro evidente que van produciendo, es posible y necesario proporcionar al adulto mayor una serie de cuidados para mantenerlo en buenas condiciones y mejorar su calidad de vida. La meta de mejorar la calidad de vida ha cobrado una mayor importancia en promoción de la salud y es especialmente importante en relación con la satisfacción de las necesidades de las personas de edad avanzada y por tanto la satisfacción de las necesidades se realizará a través del cuidado.

Para Alzate (1998), el cuidado puede tener la connotación de fin o de medio, como fin denota adaptación de algo o del individuo y/o grupos hacia el medio social al que pertenecen, para seguir respondiendo a las múltiples exigencias de su entorno. Como medio se constituye en un proceso dinámico en el que interactúan diversos elementos para mantenerse en estabilidad dentro del continuo vida-muerte. Hablar de cuidado significa hablar de 


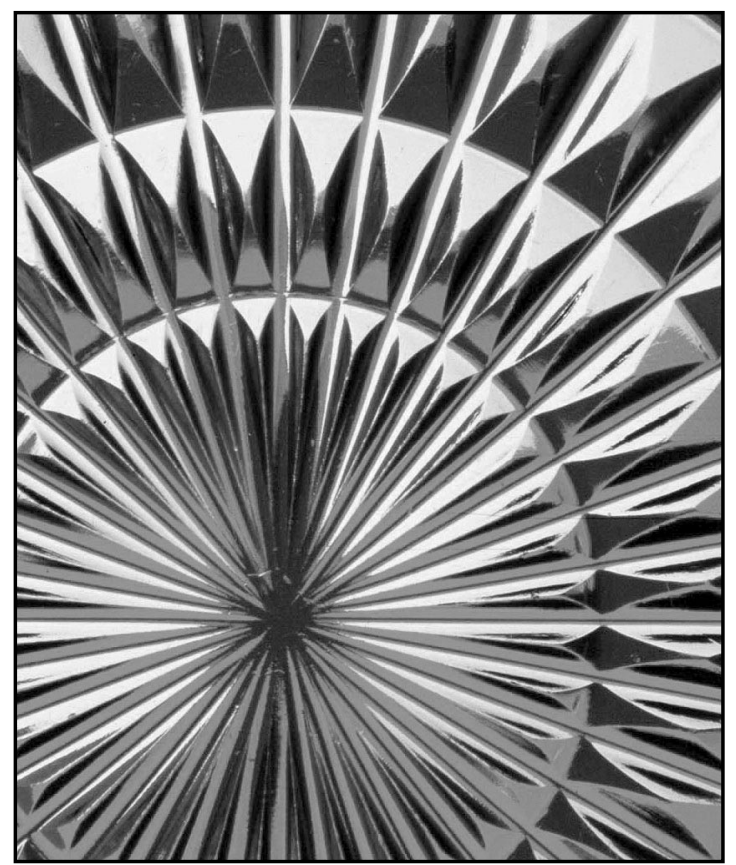

actitudes. Hablar de actitudes, porque hay elementos específicos complejos y únicos. El cuidado en si mismo es una vivencia única, tanto para quien lo recibe, como para quien lo brinda. Si en verdad quien cuida entiende el significado de la palabra cuidado o de cuidar, sabrá que nunca repetirá la experiencia de cuidar, aún en circunstancias semejantes. El valor del cuidado y de cuidar implica un nivel más alto del espíritu del ser. Cuidar involucra un compromiso ético o moral hacia la protección de la dignidad humana y a la conservación de la humanidad.

Natividad Pinto Afanador define el cuidado en casa como un conjunto de acciones que se mueven en una gama que va desde el cuidado a corto plazo hasta el cuidado a término largo; acompañado de servicios de soporte social que suplen, complementan o sustituyen el cuidado institucional.

Watson (1988), menciona que es fundamental integrar la relación transpersonal paciente-cuidador, ya que ejemplifica el proceso de cuidado humano a humano, en escenarios diferentes: institucionalizado otorgado por personal de salud, o no institucionalizado, en el hogar, donde el cuidador puede ser un integrante de la familia.

Al respecto, Shamansky (1988), refiere que el cuidado en casa es un conjunto de acciones que se mueven en una gama que va desde el cuidado a corto plazo hasta el cuidado a término largo; acompañado de servicios de soporte social que suplen, complementan o sustituyen el cuidado institucional.

Humphrey (1988), cita el concepto de cuidado en casa dado por Friedman así: "es la provisión del cuidado en el hogar para una persona que esta enferma o discapacitada al alcance de el o de ella, que no es capaz de funcionar de alguna manera como una persona normalmente saludable en nuestra sociedad". Este cuidado puede ser temporal y puede abarcar desde la satisfacción total de necesidades básicas o solamente las de apoyo educativo (orientación para la preservación del estado de salud por parte de la familia).

Para fines de este estudio delimitaremos al receptor del cuidado al adulto mayor, que se encuentra entre los 60 a 75 años de edad, ya que la capacidad de vivir más años no ha sido acompañada a una actitud similar para retrasar la pérdida de dependencia, sino que el aumento de la necesidad de cuidado impone un desafío cada vez mayor a las familias y la sociedad.

El cuidado de las personas mayores, ha sido una preocupación de todos los tiempos, pero en las últimas décadas el aumento en la expectativa de vida ha supuesto que cada vez más personas alcancen una edad avanzada ya que antiguamente, las personas no vivían tantos años y el periodo en el que necesitaban cuidados era más corto, las mujeres no salían a trabajar fuera de casa, hechos que les obligaba a cuidar a los niños y los ancianos, por lo tanto puede destacarse la relación de parentesco y género del cuidador y de la persona que recibe los cuidados, así como la calidad de las relaciones previas, el nivel cultural y socioeconómico del cuidador dado que históricamente el cuidado del anciano ha sido asumido por la familia y, en términos generales, continúa siéndolo hasta la actualidad.

Lo expresado con anterioridad es coincidente con las aportaciones de Egurza M. I. \& Notivol P. quienes mencionan que del 70 al $80 \%$ de los ancianos reciben atención por parte de la familia en el ámbito de su domicilio.Asimismo, Manuel Lillo Crespo (s/f), realizó una investigación bibliográfica de corte antropológico, con base en la metodo- 
logía cualitativa, con el propósito de mostrar los distintos valores bajo los cuales la vejez ha sido interpretada por diferentes culturas a lo largo de la vida, hasta nuestros días, facilitando la reflexión sobre las ocupaciones, el rol y los responsables del cuidado del senecto, describe que en las últimas décadas la población de edad avanzada ha aumentado en forma creciente en número y diversidad, requiriendo a su vez un mayor espectro de necesidades de todos los tipos y es la figura de la mujer la que esta siempre unida al rol de cuidadora, ya que es ella la que se ha ocupado de los cuidados del anciano, desde la prehistoria hasta el mundo contemporáneo, y es en esta época donde menciona el autor, que a diferencia del pasado, es cuando comenzamos a asistir a una mayor implicación del hombre en el cuidado del anciano dentro de la familia.

En otro estudio Merce. J., de la Sociedad Española de Geriatría y Gerontología (1988), reporta que un $30 \%$ de los adultos mayores reside con su familia. El $84 \%$ de los ciudadanos españoles piensa que cuidar a los mayores es obligación de los hijos, aunque sólo un $24 \%$ se muestra convencido de que, en el futuro, la atención de los ancianos seguirá corriendo a cargo de los hijos; sin embargo, cuando la necesidad de asistencia de los abuelos se vuelve permanente, un $29 \%$ de las personas entrevistadas estima que la ayuda debe prestarla la propia familia, un $37 \%$ opina que es competencia de los servicios sociales y un $29 \%$ cree que debe ser proporcionada por ambas partes.

En lo que respecta al perfil del cuidador, menciona que habitualmente, es el cónyuge quien se hace cargo de la persona que precisa cuidados, pero si quien requiere asistencia es la esposa, la situación se complica porque los varones no asumen fácilmente el rol de cuidador, y entonces es la hija la que asume este rol, y el problema de cuidar un anciano en casa es que las familias no están enseñadas a tratar al adulto mayor, lo que origina deficiencia en la calidad del cuidado que se brinda.

Kuypers y Bengston (1990) mencionan que por lo general, la familia ha intentado distintas formas de cuidado que resultaron deficitarias y se enfrenta a tener que aceptar la incapacidad del grupo para resolver por si misma esta situación. Esta aceptación la hace vulnerable, pues puede generar el sen- timiento de ser incompetente para afrontar el cuidado de cualquiera de sus miembros necesitados de ayuda, pudiendo iniciarse un "ciclo de ruptura familiar". Por lo que necesario educar a los familiares sobre la atención de los pacientes, ya que es posible aumentar el conocimiento sobre la atención a pacientes dependientes y sus cuidadores. Lo anterior con el propósito de que el cuidado que recibe en el hogar sea de calidad.

Por lo tanto, se reconoce que los servicios de salud enfrentan actualmente un nuevo reto que es el atender las necesidades de este sector de la población, debido a su rápido crecimiento, lo que ha traído como consecuencia efectos en la demanda del cuidado; donde no solo afecta a los servicios de salud si no a la sociedad, es decir "si no estamos involucrados todos, desde los mas pequeños hasta los ancianos, y no aceptamos esta etapa como un proceso natural, las cosas no saldrán bien" (Rodríguez, 2001), pues es necesario considerar que la familia es el principal cimiento de la sociedad, de donde surge el apoyo y la educación fundamentales para el bienestar.

\section{METODOLOGÍA.}

Por lo anterior, decidimos investigar "La calidad del cuidado del adulto mayor en escenario no institucionalizado" con el propósito de determinar la calidad del cuidado que recibe el adulto mayor en su domicilio; valorando la calidad del cuidado, a través de aspectos como la Percepción del Cuidado, capacidad física y Prácticas de auto cuidado.

Para cumplir con el objetivo de la investigación, se realizo un estudio observacional, transversal descriptivo. Donde la población de estudio correspondió a personas mayores de 60 años de edad, habitantes de la colonia la Libertad de Ciudad Victoria Tamaulipas, integrantes de un Grupo de Ayuda Mutua (GAM) de adultos mayores que se reúnen en casa de la presidenta de la colonia. El total de integrantes del GAM es de 40 personas, y se seleccionaron a aquellos que cumplieron el criterio de inclusión: adulto mayor de 60 a 75 años de edad (30 personas).

Se elaboró un instrumento para la obtención de datos que contiene 28 reactivos, correspondientes a las variables de la percepción del cuidado, como 
son: aspectos de la percepción del cuidado, aspectos físicos y los aspectos de la práctica de auto cuidado, el cual fue validado antes de su aplicación.

El plan de trabajo de campo se dividió en dos partes, la primera consistió en obtener el consentimiento informado de los participantes y la segunda parte en la aplicación del instrumento. Con la información obtenida se elaboró una base de datos con el paquete estadístico para las ciencias sociales (Statistical Package for the Social Science), SPSS 11.0 (Norusin, 1994); el análisis estadístico fue descriptivo, se obtuvieron frecuencias, media, mediana y moda.

\section{RESULTADOS}

\section{Aspectos demográficos}

Los resultados obtenidos de la encuesta aplicada a una muestra de 30 personas mayores de 60 años, indican que en la variable de edad, la media de edad es de 68 años y una desviación estándar de 5.89 , con un rango de 60 a 75 años.

El $80 \%$ de las personas encuestadas son del género femenino, restando el $20 \%$ de género masculino.

Respecto a la atención médica, el $86.7 \%$ mencionaron estar afiliados a una institución de salud, siendo el Seguro Popular el que representa el mayor porcentaje con $40 \%$, el Instituto Mexicano del Seguro Social con $33.3 \%$ y el $10 \%$ señaló que no cuenta con servicio de atención médica.

El $90 \%$ de las personas encuestadas refirieron estar enfermos actualmente, siendo las enfermedades crónico degenerativas las de mayor frecuencia con (Diabetes Mellitus e Hipertensión arterial) $73.3 \%$ y las enfermedades respiratorias el $6.7 \%$, el $10 \%$ mencionaron otros tipos de padecimientos y $10 \%$ no reportaron enfermedad alguna, el $86.7 \%$ recibe tratamiento actualmente y el $13.3 \%$ no se encuentra bajo tratamiento, los hipoglucemiantes representan el tratamiento más frecuente con el $40 \%$, siguiendo los antihipertensivos con el $30 \%$ de frecuencia, los antihistamínicos representan el $3.3 \%$ y otros tratamientos el $13.3 \%$.

Para continuar con la percepción que tiene el adulto mayor de su estado de salud, el $56.7 \%$ menciona que su estado de salud es regular, el $40 \%$ lo reporta como bueno y sólo el $3.3 \%$ lo considera muy bueno, cuestionando sobre si posee la Cartilla
Nacional del Adulto Mayor, el 90\% responde que no cuenta con ese documento.

\section{Percepción del cuidado}

El $36.7 \%$ de estos adultos, ha requerido el cuidado en casa en algunas ocasiones, el $26.7 \%$ mencionan haberlo necesitado casi siempre, el $23.3 \%$ respondió no haberlo requerido nunca y el $13.3 \%$ haberlo requerido siempre.

Otro de los cuestionamientos realizados fue saber si el cuidado en casa se lo brindo una mujer, a lo que la mayoría de los encuestados que representan el $36.7 \%$ respondió nunca, el 30\% mencionó casi siempre y siempre respectivamente haber recibido atención de una mujer, el $43.3 \%$ de los casos siempre tienen confianza en los consejos de salud que les proporciona la persona que los cuida en casa, mientras el $26.7 \%$ y $23.3 \%$ consideraron que casi siempre y siempre respectivamente a ese cuestionamiento.

Respecto a la satisfacción del cuidado que recibe en casa, 15 personas, representando el $50 \%$ considera estar siempre satisfecho, el $23.3 \%$ casi siempre, y sólo 7 (23.3\%) personas consideraron no estar satisfechas con ese cuidado otorgado.

El $43.3 \%$ de los adultos mayores encuestados consideran que el cuidado que reciben en casa, siempre contribuye a mantenerlos sanos, sin embargo el $23.3 \%$ y $20 \%$ refieren que casi siempre y algunas veces, respectivamente, contribuyen a éste propósito. La mayoría de las personas (40\%) prefiere quedarse siempre en casa y recibir el cuidado ahí, que ir a una institución de salud, el $16.7 \%$ refieren que algunas veces y casi siempre prefieren quedarse a recibir el cuidado en casa.

El 33.3\% señala que nunca recibe información de su familia sobre cómo cuidar su salud y con un mismo porcentaje, que casi siempre recibe éste tipo de información.

\section{Capacidades físicas}

En el aspecto de capacidad física, 50\% informa poder realizar siempre actividades físicas, como son subir y bajar escaleras, ir de compras, etcétera, el $26.7 \%$ lo hace casi siempre y el $10 \%$ indica no hacerlo.

El $73.3 \%$ considera ser siempre capaz de cuidar de si mismos, mientras el $13.3 \%$ refiere poder 


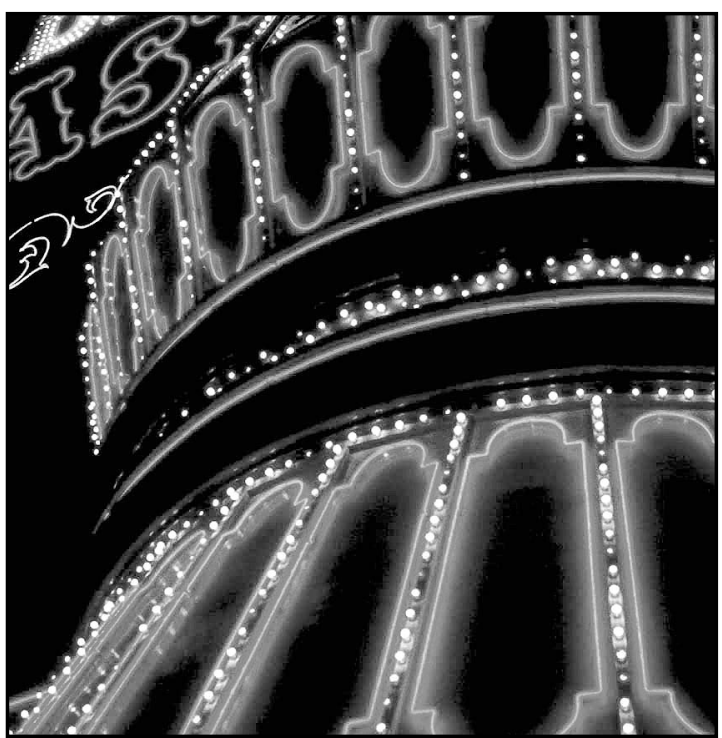

hacerlo casi siempre, el 10\% algunas veces y sólo el $3.3 \%$ no poder cuidar de su persona.

Se les cuestionó sobre si puede utilizar el transporte público por si mismo, a lo que el $70 \%$ respondió siempre hacerlo, sin embargo el $13.3 \%$ de los encuestados indica que no puede realizar ésta acción, el $10 \%$ y $6.7 \%$ señaló hacerlo casi siempre $\mathrm{y}$ algunas veces, respectivamente.

El 76.7\% señala que su estado de salud les permite realizar siempre actividades cotidianas por si mismos, asimismo el $13.3 \%$ responde casi siempre estar en condiciones de realizarlas, el $6.7 \%$ algunas veces y sólo 1 persona (3.3\%) no poder hacerlo.

A la pregunta si puede ayudar en el cuidado de sus nietos o de otros menores, la respuesta obtenida fue igualmente del $30 \%$ quienes respondieron nunca y algunas veces, casi siempre el $33.3 \%$, mientras los que siempre pueden ayudar con esta actividad representan el $6.7 \%$.

De las personas entrevistadas, 16 casos (53.3\%) indican ser capaces de poder llevar un control adecuado de sus enfermedades cuando éstas se presentan, el $30 \%$ señala poder hacerlo casi siempre, el $13.3 \%$ algunas veces y sólo 1 $(3.3 \%)$ persona no poder hacerlo nunca.

\section{Capacidades de auto cuidado}

Con la finalidad de conocer las capacidades de auto cuidado, una de las interrogantes fue saber si se realiza valoración de la presión arterial a lo que el $46.7 \%$ indica hacerlo casi siempre, el $26.7 \%$ realizarlo siempre, el $20 \%$ algunas veces y sólo $6.7 \%$ no practicarse este tipo de valoración.

En relación a la diabetes mellitus, el 36.7\% señala practicarse la prueba de control de glicemia capilar algunas veces, el 30\% hacerlo siempre, el $23.3 \%$ casi siempre y el $10 \%$ informa no hacerlo.

$\mathrm{Al}$ preguntárseles sobre el control adecuado de peso, 33.3\% señaló llevar algunas veces este tipo de control; quienes lo realizan casi siempre, representan el $26.7 \%$, quienes lo efectúan siempre son el $20 \%$ y otro $20 \%$ no llevan este tipo de control.

El 76.7\% no acude a atención dental, el 16.7\% lo realiza algunas veces y el $3.3 \%$ practica este cuidado casi siempre y siempre respectivamente.

El $66.7 \%$ nunca acude a atención médica a valorar su estado de salud, cuando no está enfermo, el 20\% siempre acude a valorar su estado de salud aun no estando enfermo y el $3.3 \%$ casi siempre valora su estado de salud en ausencia de enfermedad.

En relación si es capaz de administrar o manejar sus propios medicamentos y tomarlos a la hora prescrita, el 53.3\% señala hacerlo siempre, el 3.3\% no hacerlo nunca y el 20 y $23.3 \%$ realizarlo casi siempre y algunas veces correspondientemente.

El $73.3 \%$ indica no acudir a una unidad de salud a demandar atención preventiva cuando se lleva a efecto la Semana de Salud para la Gente Grande, y sólo el 3.3\% señala hacerlo siempre.

En cuanto a la actividad física, entendiendo ésta como el ejercicio para la salud, $20 \%$ señalan hacerlo algunas veces, el $43.3 \%$ no hacerlo nunca y sólo $36.7 \%$ realizarla siempre.

El $43.3 \%$ se aplica vacunas de acuerdo a su edad algunas veces, el $20 \%$ no aplicarlas y con el mismo porcentaje se encuentran las que siempre se aplican dichos biológicos.

Para la detección oportuna de cáncer cérvico uterino y de mama, el $41.6 \%$ no se lo ha realizado nunca, mientras el $33.3 \%$ algunas veces, el $12.5 \%$ refiere hacerlo casi siempre y otro tanto, siempre.

De las 6 personas de género masculino entrevistadas, 5 de ellos (83.3\%) refiere no realizarse nunca detección de hiperplasia prostática y sólo un caso $(16.6 \%)$ señala realizarlo algunas veces.

En relación a su participación en actividades de educación para la salud y fomento de estilos de 
vida saludables que promueve el grupos de ayuda mutua al que pertenecen; el $36.7 \%$ menciono participar siempre en este tipo de actividades, un $30 \%$ menciona que nunca participa y el $23.3 \%$ señala participar casi siempre y el $10 \%$ algunas veces participar.

Más de la mitad de los casos $(63.3 \%)$ asevera ser todavía una persona útil, el $20 \%$ responde serlo casi siempre, $13.3 \%$ algunas veces y sólo una persona $(3.3 \%)$ no cree serlo.

El $70 \%$ de estas personas mantiene siempre buenas relaciones con amigos y vecinos, el $20 \%$ indican mantenerlas casi siempre.

Respecto a la cuestión de salir a distraerse, ya sea sólo, con la familia o amigos, el $43.3 \%$ afirma hacerlo algunas veces, el $30 \%$ asevera distraerse siempre, el $16.6 \%$ casi siempre y el $10.0 \%$ no salir nunca a distraerse.

\section{Discusión y análisis de los datos}

En base en los resultados obtenidos, se observa una feminización de la población, razón por la cual de los $36.7 \%$ (11) adultos mayores que han requerido el cuidado en casa, solo en el $30 \%$ (9) el cuidador ha sido una mujer. Lo anterior coincide con lo que describió la Sociedad Española de Geriatría y Gerontología (1988) en lo que respecta al perfil del cuidador, menciona que habitualmente es el cónyuge quien se hace cargo de la persona que precisa de los cuidados. El 50\% de las personas que reciben cuidado en casa esta satisfecho con los cuidados recibidos y el $43.3 \%$ refirió que estos cuidados contribuyen al mejoramiento de su salud.

En relación al nivel de salud, se identificó que las enfermedades crónico degenerativas presentan una elevada prevalencia, y precisamente este grupo de enfermedades son objeto de programas y acciones especificas de promoción y fomento de la salud; y a este respecto llama la atención que el $86.7 \%$ de los entrevistados cuenta con atención médica de forma permanente, sin embargo el $90 \%$ de ellos no cuenta con Cartilla Nacional de Salud, lo que significa que no llevan un registro adecuado de las detecciones de estos padecimientos (cuando se los realiza), razón por la cual probablemente se diagnostiquen estas enfermedades en etapa tardía, esto debido a que un $33.3 \%$ señalo que nunca reciben información de la familia sobre como cuidar su salud y este es un factor importante como condicionante del cuidado.

Por otro lado, el $56.7 \%$ mencionó que su estado de salud es regular, lo que les permite tener capacidad de cuidar de si mismos $(70 \%)$, y realizar las actividades cotidianas (76.7\%), así como llevar un control adecuado de sus enfermedades, cuando estas se presentan $(53.3 \%)$, por lo que se consideran personas útiles aún $(63.3 \%)$ con capacidades para realizar actividades de la vida diaria.

En lo que respecta a las capacidades de auto cuidado, según los datos obtenidos, los adultos mayores no realizan acciones que contribuyan a la preservación de su salud y sólo acuden a la atención medica cuando presentan un problema de salud; y pese a que presentan enfermedades crónico degenerativas y pertenecen a un GAM , asisten a las reuniones del grupo de autoayuda, sin embargo no participan en las actividades de promoción de estilos de vida saludables que se les recomiendan ya que solo el $36.7 \%$ de los entrevistados refirió participar en estas actividades, por lo que consideramos que el propósito del GAM, que es inducir en el adulto mayor cambios permanentes en el estilo de vida y que adquiera habilidades y destrezas para su auto cuidado, mediante el constante contacto con el equipo de salud en el GAM no se cumple, ya que los pacientes asisten a las reuniones pero de forma pasiva; y estas reuniones no tienen un impacto positivo en las acciones que realizan para la preservación del estado de salud.

De acuerdo con la información obtenida podemos concluir que el cuidado que recibe el adulto mayor en su domicilio, es cuidado a corto plazo, con acciones que complementan o sustituyen el cuidado Institucional ya que este solo abarca la satisfacción de necesidades básicas, excluyendo las de apoyo educativo (orientación para la preservación del estado de salud por parte de la familia).

Por lo que consideramos, que este cuidado contribuye solamente en el mantenimiento de las capacidades físicas del adulto mayor, o ayudarle a realizar actividades de la vida diaria y controlar el régimen diario de medicamentos recetados., sin embargo no podemos afirmar que esto contribuya a su calidad de vida, ya que de acuerdo con la OMS (1994), el concepto de calidad de vida, no solo abarca salud física, si no también el estado psico-social. 
Por lo que concluimos, con la opinión del Dr. Merce J., quien menciona que el problema de cuidar un anciano en casa es que las familias no están enseñadas a tratar al adulto mayor, lo que origina deficiencia en la calidad del cuidado. Si se quiere realmente conseguir que las personas mayores alcancen la edad avanzada con calidad de vida, son necesarias intervenciones de prevención con materiales de apoyo dirigidos no solo al adulto mayor, sino también a los cuidadores, con el propósito de prepararlos para brindar cuidado de calidad al adulto mayor cuando lo requiere.

\section{BIBLIOGRAFÍA}

- Alzate, M. (1998). “Gerencia del cuidado de enfermería”. En Dimensiones del cuidado. Grupo de Cuidado, Facultad de Enfermería. Universidad Nacional de Colombia. Unibiblos. Santa fe de Bogotá, D.C. Colombia.103 págs.

- Bazo M. T. (1998). Vejez dependiente, políticas y calidad de vida.

- Caballero A,, Naranjo A. M., Fong G. Y., Ministerio de Salu Pública Policlínico "Frank País García”, Distrito José Martí, Santiago de Cuba, Cuba.

- Camejo T. R., Álvarez E. T \& Pérez N. A. (s/f). Salud en la Tercera Edad y Calidad de Vida.

- Crespo L.M. (s/f). Antropología de los cuidados en el anciano: Evolución de los valores sociales sobre la vejez a través de la historia. Alicante España.

- Egurza M. I. \& Notivol P., (1999) La enfermera en la asistencia sanitaria al anciano. Anales Vol. 22, N -1.

- Hankwitz, P. (1991). Asegurando calidad del cuidado en el hogar. Clinics in Geriatric Medicine. Vol.7 No. 4 p. 647.

- Humphrey, C. (1988). The Home as a Setting for Care. Clarifying the Boundaries of Practice. Nursing Clinics of North America. Vol. 23. No. 2.
- Kuypers J., Bengston (1990). Toward undersanding healt in older familias impacted by catastrophic illnes. En T.H. Brubaker (Ed), Family relationships in later life, Newbury Park: Sage publications.

- Lehr U. (1993). Correlatos Psicosociales de la Longevidad. Conferencia en Bogotá.

- Marín G. \& Ángeles V. P. (1994). La mujer y el cuidado de ancianos.

- Márquez, Susana, "Cuidar con cuidado". En Enfermería Clínica, Vol. 5, núm.6. pág. 266. Policopiado.

- Mercè, J., (1988) Sociedad Española de Geriatría y Gerontología. España.

- Organización Mundial de la Salud. (1994).

- Papalia, Wendkos y Duskin. (2001) Desarrollo Humano. $8^{\circ}$. Edición. Ed. Mc Graw Hill Interamericana.

- Pinto, A. N. (2000). Hacia un Modelo de Cuidado en el Hogar. EN Cuidado y Práctica de Enfermería. Bogotá: Facultad de Enfermería, Universidad Nacional de Colombia, 2000. Págs. $57-72$.

- Rodríguez E. (2001) Cuidadores Informales: Necesidad y ayudas. Revista Rol de Enfermería, 24 (3)

- Ruiz D. E., Quintero. (1999). G. CV y Envejecimiento. En: Rocabruno JC. Tratado de Gerontología y Geriatría Clínica. La Habana. Editorial Academia; 1999:128-136.

- Rubalcava R.M. (1999) La situación Demográfica en México. Consejo Nacional de Población. México D.F.

- Shamansky, S. (1988). Providing Home Care Services in For - Profit Environment-Nursing Clinics of North America. Vol. 23 No. 2. p. 388.

- Watson, J. (1988). Cuidado humano en enfermería. Enfermería: ciencia humana y cuidado humano. Una teoría de Enfermería. National League for Nursing. p. 28.

- Watson J. (2001) Assessing and measuring caring in nursing and healt sciences. New York: Sringer Publishing Company.

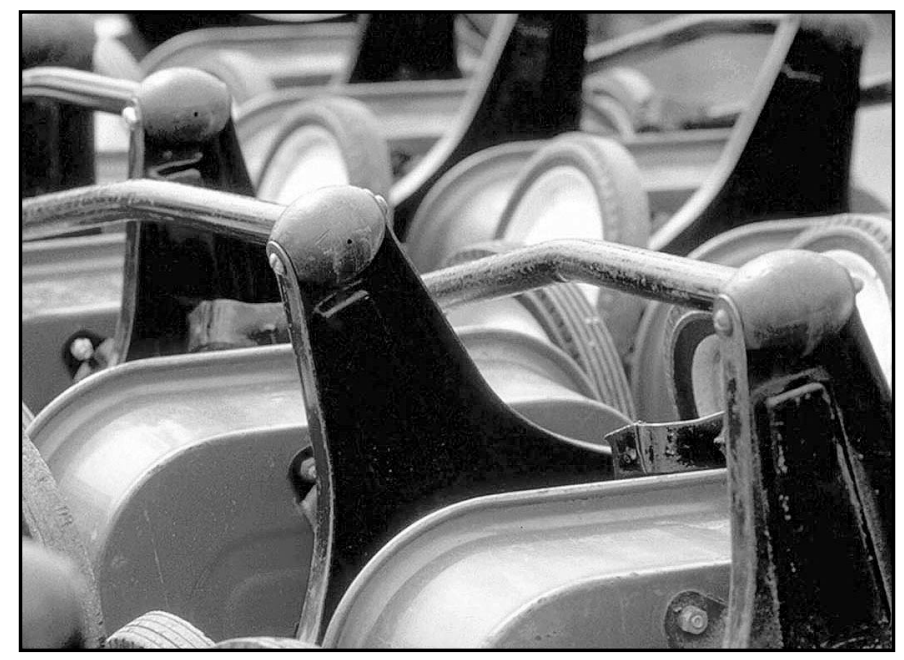

